\title{
Exploring The Current Management Idiopathic Intracranial Hypertension, And Understanding The Role Of Dural Venous Sinus Stenting
}

This article was published in the following Dove Press journal: Eye and Brain

\author{
Sam P Gurney' \\ Sateesh Ramalingam (iD) ${ }^{2}$ \\ Alan Thomas ${ }^{2}$ \\ Alex J Sinclair (iD ${ }^{3-5}$ \\ Susan P Mollan (D) \\ 'Birmingham Neuro-Ophthalmology, \\ Queen Elizabeth Hospital, University \\ Hospitals Birmingham, Birmingham BI5 \\ 2WB, UK; ${ }^{2}$ Neuroradiology Department, \\ Queen Elizabeth Hospital, University \\ Hospitals Birmingham, Birmingham BI5 \\ 2WB, UK; ${ }^{3}$ Metabolic Neurology, \\ Institute of Metabolism and Systems \\ Research, College of Medical and Dental \\ Sciences, University of Birmingham, \\ Birmingham BI5 2TT, UK; ${ }^{4}$ Centre for \\ Endocrinology, Diabetes and Metabolism, \\ Birmingham Health Partners, Birmingham \\ BI5 2TH, UK; ${ }^{5}$ Department of \\ Neurology, University Hospitals \\ Birmingham NHS Foundation Trust, \\ Queen Elizabeth Hospital, Birmingham \\ BI5 2WB, UK
}

Correspondence: Susan P Mollan Birmingham Neuro-Ophthalmology, Queen Elizabeth Hospital, University Hospitals Birmingham, Birmingham BI5 2WB, UK

Tel +44 I2137/6912

Email susan.mollan@uhb.nhs.uk

\begin{abstract}
Idiopathic Intracranial Hypertension (IIH) is a debilitating disorder characterised by raised intracranial pressure (ICP), papilloedema with the potential risk of permanent visual loss, and headaches that are profoundly disabling and reduce the quality of life. The first consensus guidelines have been published on investigation and management of adult IIH and one key area of uncertainty is the utility of dural venous sinus stenting for the management of headache and visual loss. There are an increasing number of series published and to help understand the successes and complications. During a patient physician priority setting, the understanding of the best type of intervention to treat IIH was assigned to the top 10 of most desired research questions for the disease. Ultimately randomised clinical trials (RCTs) in neurovascular stenting for IIH would be instructive, as the literature to date may suffer from publication bias. Due to the increasing incidence of IIH, there is no better time to systematically investigate interventions that may reverse the disease process and achieve remission. In this review we discuss the pathophysiology of IIH in relation to venous sinus stenosis, the role of venous sinus stenting with a review of the relevant literature, the advantages and disadvantages of stenting compared with other surgical interventions, and the future of stenting in the treatment of IIH.
\end{abstract}

Keywords: pseudotumor cerebri, raised intracranial pressure, venous sinus stenosis, neurovascular stenting, headache, papilloedema

\section{Introduction}

Idiopathic intracranial hypertension (IIH) is a disorder of unknown aetiology, primarily affecting women of childbearing age with raised body mass index (BMI), characterised by signs and symptoms of raised intracranial pressure (ICP). ${ }^{1} \mathrm{IIH}$ is a diagnosis of exclusion, typically made using the modified Dandy criteria, ${ }^{2}$ requiring the presence of raised ICP $\left(>25 \mathrm{~cm} \mathrm{H}_{2} \mathrm{O}\right.$ on lumbar puncture, performed in the lateral decubitus position), with papilloedema typically present, and normal cerebrospinal fluid (CSF) profile and neuroimaging. In a retrospective case review, the incidence of IIH was reported as 1.56 per 100,000 in the general population, increasing to 11.90 per 100,000 in obese young women. ${ }^{3}$ A recent paper highlighted the growing epidemic of IIH, most notably in areas of social deprivation (thereby mirroring trends in obesity); over their 14-year study period, incidence in the general UK population increased by greater than $100 \%$ to 4.7 per $100,000 .{ }^{4}$ With more and more patients presenting, there is an unmet management need in $\mathrm{IIH}$, namely safe and effective therapies. 
The major risk factor for IIH is obesity, ${ }^{5}$ with truncal fat mass being associated with lumbar puncture opening pressure. ${ }^{6}$ Hence, management strategies focus on disease modification through weight loss, although this by lifestyle alone is difficult to achieve. The underlying aetiology is not fully understood ${ }^{1,5}$ but neuroendocrine metabolic pathways are being studied, ${ }^{7}$ and recently a unique signature of androgen excess in IIH has been implicated and provides evidence that androgens can modulate CSF secretion via the choroid plexus. ${ }^{8}$ Intriguingly, women with IIH appear to have a 2-fold increase in the cardiovascular disease risk, over and above age, gender and BMI matched controls, providing further evidence that this may well be a neurometabolic disease. $^{9}$

The clinical presentation varies but symptoms classically include headache; however, our understanding of the traditional phenotype of raised ICP headache has progressed and often those with IIH have headaches that fulfil the criteria for migraine. ${ }^{10,11}$ Other common symptoms include pulse synchronous tinnitus, transient visual obscurations, double vision (from unilateral or bilateral 6th nerve palsy), reduction in visual acuity or visual field, and neck pain ${ }^{1,12}$ Previously termed as benign intracranial hypertension, IIH was renamed to reflect the fact that the condition may be associated with severe morbidity, most commonly permanent visual loss, affecting up to $24 \%$ of patients in early studies ${ }^{13}$ and a reduced quality of life which is linked to headache morbidity. ${ }^{14}$

Following the success of collaborative patient-clinician research $^{15}$ and the clinical uncertainty highlighted in managing $\mathrm{IIH},{ }^{16}$ the patient group founded a James Lind Alliance Priority Setting Partnership for adult IIH. $^{17}$ Understanding of the best type of intervention to treat IIH was placed within the top 10 priorities for research ${ }^{17}$ highlighting the interest in modern intervention such as dural venous sinus stenting for IIH.

\section{Medical Management Of IIH}

Consensus guidance published in 2018 stated uncertainties in all aspects of IIH management. ${ }^{16}$ Indeed, a Cochrane review of interventions for $\mathrm{IIH}^{18}$ judged the literature in medical management of IIH with acetazolamide, the preferred drug, to be of low quality, despite two randomised controlled trials (RCT). ${ }^{19,20}$ However, it has been observed that management with weight loss was effective in the treatment of IIH. For example, a prospective study ${ }^{21}$ has demonstrated that a very low-calorie diet resulting in significant weight loss $(15.3 \pm 7.0 \%$ of body weight) significantly lowered ICP and led to a significant improvement in papilloedema, vision and headache outcomes. There is an ongoing multicentre, randomised controlled trial ${ }^{22}$ designed to assess if weight loss through bariatric surgery is a more effective sustainable treatment for IIH than lifestyle modification through a community weight management program.

Medical management to reduce ICP is typically with acetazolamide, a carbonic anhydrase inhibitor, which is thought to act by reducing CSF secretion at the choroid plexus. Two RCTs have been carried out to study the effect of acetazolamide versus placebo (with both groups receiving weight-reduction diet and lifestyle modification) for the treatment of IIH. Ball et al ${ }^{19}$ showed a small benefit for the use of acetazolamide at 12 months, with a reduction in headache, vision loss, transient visual obscurations, and improvement in contrast sensitivity. They did not find evidence of reduction in papilloedema, headache severity, improvement in visual acuity, or visual field parameters. Wall et $\mathrm{al}^{20}$ showed (in the treatment group at 6 months) an improvement in visual field, papilloedema, quality of life, weight loss, and CSF opening pressure. The authors of a Cochrane review based on these two RCTs were however unable to recommend or reject acetazolamide for the treatment of IIH. ${ }^{18}$ One study suggested non-inferiority of topiramate, an anti-epileptic and migraine prophylaxis, versus acetazolamide. ${ }^{23}$ The proposed advantage of this drug is its combined effects of carbonic anhydrase inhibition, migraine prophylaxis, and appetite suppression.

A recent in vivo study investigated the commonly cited medications for IIH at both clinical (single equivalent human dose) and high (daily equivalent human dose) namely, subcutaneous acetazolamide $1 \mathrm{~g}$ and $4 \mathrm{~g}$; Topiramate $50 \mathrm{mg}$ and $200 \mathrm{mg}$; and oral preparations of Topiramate $200 \mathrm{mg}$ and Acetazolamide $4 \mathrm{~g}$. At clinical and high doses, subcutaneous administration of topiramate significantly lowered ICP over $2 \mathrm{hrs}$ to $68.6 \pm 2.0 \%$ of baseline which was a $32 \%$ reduction $(p<0.001)$ and by $79.2 \pm 7.5 \%$ of baseline, which was a $21 \%$ reduction $(p<0.05)$, respectively, compared to the control. Of note, there was no significant reduction in ICP noted with equivalent to human doses of acetazolamide, amiloride, furosemide, or octreotide. Oral administration of topiramate lowered ICP, and over the first two hours, topiramate significantly reduced ICP compared to baseline (22\% reduction, $p<0.05$ ). Compared to placebo, acetazolamide did not significantly lower ICP over the same study period. ${ }^{24}$ Human physiological studies would be further informative in assessing ICP levels in response to the common medications used. On the horizon may be more medical options, including $11 \beta$ Hydroxysteroid Dehydrogenase Type 1 inhibitors. ${ }^{25}$ 


\section{Surgical Management For IIH}

In less than $10 \%$ of $\mathrm{IIH}^{4}$ present with rapidly progressive loss of visual function (termed fulminant IIH) and in whom an acute reduction in ICP is required to preserve vision, surgical interventions are necessary. ${ }^{16,26}$ Such options include CSF diversion with the commonest surgeries being a ventriculoperitoneal shunt or lumboperitoneal shunt, or optic nerve sheath fenestration (ONSF).

The disadvantages of any intervention are that they can be invasive, carry inherent risks to the patient and may not relieve headache symptoms. Shunt revision surgeries are very common, with a third of patients requiring multiple revision surgeries. ${ }^{27}$ Ventriculo-peritoneal shunts are preferred ${ }^{16,26}$ due to the reported lower revision rates compared to lumboperitoneal shunts (1.8 versus 4.3 revisions per patient, respectively) ${ }^{28}$ It is important to counsel patients that ventriculo-peritoneal shunt insertion leads to a temporary driving restriction in some countries such as the UK. ${ }^{16}$

ONSF surgery is another option, particularly for asymmetric papilloedema or when visual symptoms predominate. It carries the risk of severe complications such as loss of vision, as well as diplopia and pupillary dysfunction. ${ }^{29}$

\section{Dural Venous Sinus Stenting}

Venous sinus stenting was first described by Higgins et $\mathrm{al}^{30}$ in 2002 , with the technique of inserting a catheter into the internal jugular vein to direct a self-expanding stent over a guidewire across a venous sinus stenosis. In this seminal case, the dural venous pressure gradient reduced from 18 $\mathrm{mmHg}$ pre-procedure to $3 \mathrm{mmHg}$ post-procedure, with the patient reporting an improvement in their headache. Since then it has been added to the surgical armamentarium, in some centres, for IIH. Several studies (Table 1) have demonstrated seemingly good efficacy and favourable safety profile. $^{30-49}$ In this paper, we discuss the pathophysiology of IIH in relation to venous sinus stenosis, the rationale and role of venous sinus stenting with a review of the relevant literature, the advantages and disadvantages of stenting compared with other surgical interventions, and the future of stenting in the treatment of IIH.

\section{Pathophysiology Relating To Venous Sinus Stenosis}

The precise pathogenesis of IIH is still not fully understood, but clinical signs and symptoms point to raised ICP in the absence of hydrocephalus or ventriculomegaly. Therefore, the primary mechanisms are proposed to be
CSF hypersecretion, CSF outflow obstruction and, more recently, venous sinus hypertension. Other contributing factors are thought to include cerebral oedema, obesity, hormonal factors, as well as important secondary causes (for example, corticosteroids, certain antibiotics, and vitamin A) and associated disorders (such as anaemia, obstructive sleep apnoea, and renal failure). ${ }^{5,16}$

Dural venous sinus occlusion, most commonly occurring in the superior sagittal sinus (SSS) or transverse sinus (TS) secondary to thrombosis or a compressive lesion, is known to cause raised ICP and clinical features synonymous with IIH. However, partial occlusion or stenosis with resultant venous sinus hypertension is now increasingly recognised in patients with IIH. It has been hypothesised for some time that patients with IIH have anatomically different dural venous sinuses. ${ }^{50}$ A link between venous sinus hypertension and IIH was demonstrated by King et $\mathrm{al}^{51}$ in 1995 when manometry was performed on nine patients with IIH. Raised pressure in the SSS and proximal TS was found in all of them, with a mean pressure gradient of $13.3 \mathrm{mmHg}$. The reported incidence of venous sinus stenosis in patients with IIH ranges from $30 \%$ to $93 \%{ }^{38}$ compared with $6.8 \%$ among the general population. ${ }^{52}$ One imaging study ${ }^{50}$ noted bilateral venous sinus stenosis in $93 \%$ of IIH patients, compared with $7 \%$ of controls. As such, our understanding about the importance of dural venous haemodynamics in the pathophysiology of IIH has greatly increased in recent years; indeed, venous sinus hypertension has been hypothesised by some to be the cause of IIH. ${ }^{13}$ However, there is still debate as to whether dural venous sinus stenosis is the cause or consequence of refractory IIH.

CSF absorption occurs primarily via the arachnoid villi of the SSS and is determined by the following equation: CSF absorption $=\left(\mathrm{P}_{\mathrm{CSF}}-\mathrm{P}_{\mathrm{SSS}}\right) / \mathrm{R}_{\mathrm{O}}$, where $\mathrm{P}_{\mathrm{CSF}}$ is the CSF pressure, $P_{S S S}$ is the SSS pressure, and $R_{O}$ is the resistance across the arachnoid villi. ${ }^{53}$ This implies that CSF absorption (and thus ICP) are governed by the venous pressure in the SSS. ${ }^{31}$ To facilitate CSF drainage via the arachnoid granulation, CSF pressure must be higher than venous sinus pressure. The walls of the venous sinuses must therefore be rigid in order to maintain patency when surrounded by higher CSF pressure.

An alternative theory reverses this mechanistic relationship, suggesting that rather than being causative, venous sinus stenosis may occur in susceptible regions of the sinuses as a result of raised ICP. Evidence to support this includes studies showing reversal of venous sinus 


\begin{tabular}{|c|c|c|c|c|c|c|c|c|c|c|c|c|c|c|}
\hline 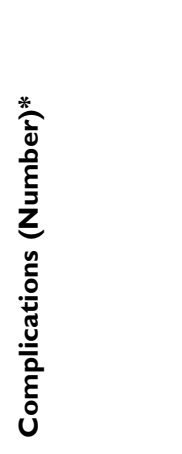 & 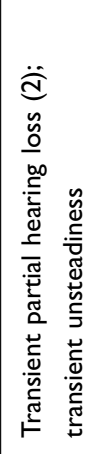 & 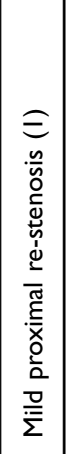 & \begin{tabular}{|l}
0 \\
$\check{0}$ \\
$z$ \\
$z$
\end{tabular} & 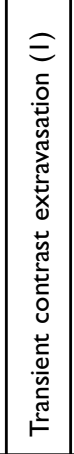 & 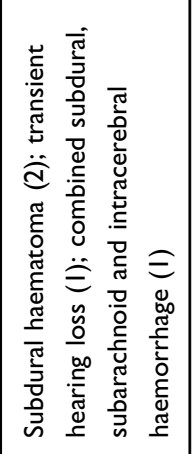 & 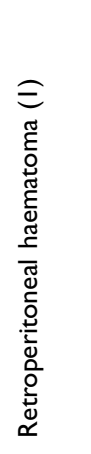 & $\mid \begin{array}{c}0 \\
\stackrel{0}{0} \\
z\end{array}$ & $\begin{array}{l}\text { ¿ } \\
\text { Oे } \\
z\end{array}$ & 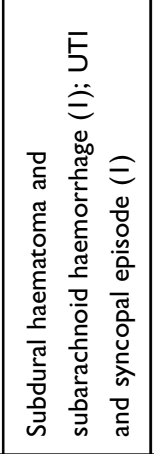 & $\begin{array}{l}0 \\
\check{0} \\
z\end{array}$ & 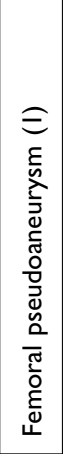 & 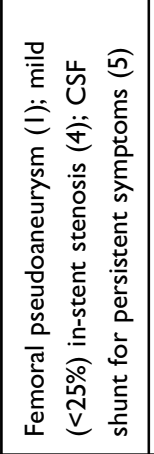 & 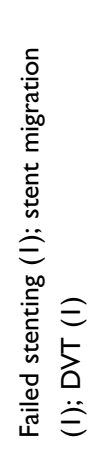 & 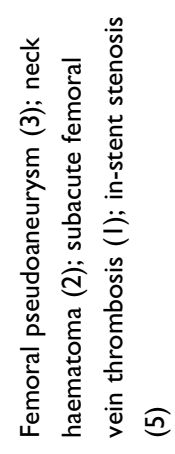 \\
\hline 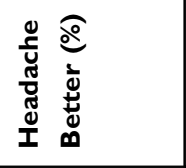 & 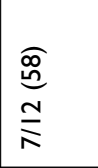 & \begin{tabular}{|l}
$\frac{8}{6}$ \\
$\frac{7}{2}$
\end{tabular} & $\begin{array}{c}\widehat{\Phi} \\
\stackrel{\infty}{\infty} \\
\frac{\circ}{\infty} \\
\end{array}$ & $\begin{array}{l}\stackrel{\widehat{o}}{\varrho} \\
\stackrel{\circ}{o}\end{array}$ & 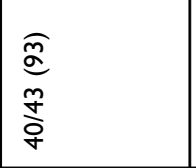 & $\begin{array}{l}\stackrel{\widehat{o}}{\varrho} \\
\stackrel{n}{\underline{a}}\end{array}$ & 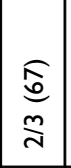 & 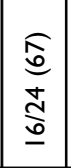 & 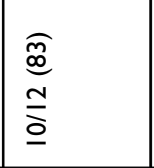 & $\begin{array}{l}\widehat{\tilde{\alpha}} \\
\stackrel{\infty}{\tilde{c}} \\
\underline{\bar{o}}\end{array}$ & $\begin{array}{l}\text { Ê } \\
\stackrel{\omega n}{0} \\
\underline{0}\end{array}$ & 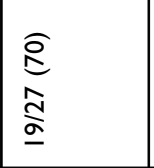 & $\begin{array}{l}\widehat{D} \\
\stackrel{0}{n} \\
\stackrel{\infty}{0}\end{array}$ & $\begin{array}{l}\underset{\infty}{\infty} \\
\hat{m} \\
\frac{\hat{m}}{m}\end{array}$ \\
\hline 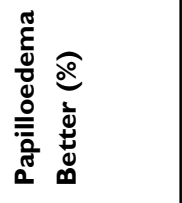 & $\begin{array}{l}\widehat{\bar{E}} \\
\hat{\mathrm{N}}\end{array}$ & $\left|\begin{array}{l}\hat{f} \\
\hat{m} \\
\hat{n}\end{array}\right|$ & 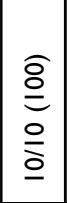 & $\begin{array}{l}\widehat{\partial} \\
\stackrel{\delta}{\sigma} \\
\frac{\sigma}{\sigma}\end{array}$ & 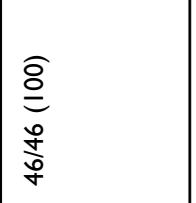 & $\stackrel{\alpha}{Z}$ & $\begin{array}{l}\underset{\delta}{\stackrel{o}{=}} \\
\frac{m}{m}\end{array}$ & 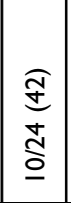 & $\begin{array}{l}\frac{f}{0} \\
0 \\
\frac{0}{3}\end{array}$ & $\begin{array}{l}\widehat{\Xi} \\
\stackrel{\Xi}{\Xi} \\
=\end{array}$ & 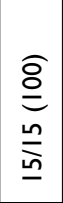 & 号 & $\begin{array}{l}\underset{0}{\infty} \\
\stackrel{\infty}{=}\end{array}$ & $\begin{array}{l}\overparen{\mathscr{D}} \\
\stackrel{\infty}{\infty} \\
\stackrel{\infty}{\sim} \\
\stackrel{\gamma}{\sigma}\end{array}$ \\
\hline 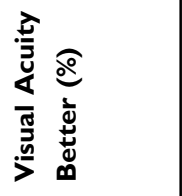 & 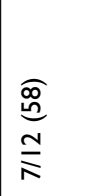 & 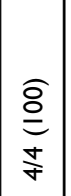 & $\mid \begin{array}{l}\widehat{a} \\
\frac{0}{\alpha}\end{array}$ & 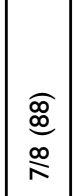 & 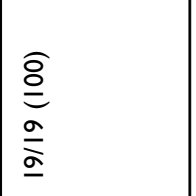 & $\stackrel{\alpha}{Z}$ & $\begin{array}{l}\underset{\delta}{\sigma} \\
\stackrel{m}{m}\end{array}$ & 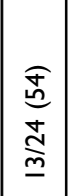 & 号 & 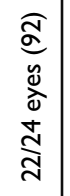 & $\begin{array}{l}\hat{\sigma} \\
\stackrel{m}{N}\end{array}$ & 号 & 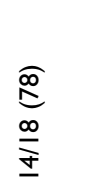 & 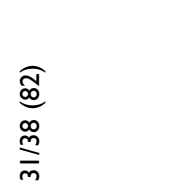 \\
\hline 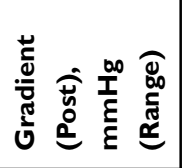 & 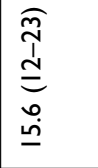 & $\widehat{\bar{c}}$ & $\frac{\mathscr{c}}{Z}$ & $\begin{array}{l}\underset{\mathfrak{d}}{d} \\
\stackrel{m}{=} \\
=\end{array}$ & $\begin{array}{l}\frac{\mathrm{v}}{\mathrm{v}} \\
\frac{\mathrm{a}}{\mathrm{d}} \\
\end{array}$ & $\stackrel{\alpha}{Z}$ & $\begin{array}{l}\frac{1}{p} \\
\stackrel{p}{N} \\
\hat{m}\end{array}$ & $\stackrel{\circ}{\stackrel{2}{*}}$ & $\begin{array}{l}\hat{T} \\
0 \\
0 \\
0 \\
\text { in }\end{array}$ & $\begin{array}{l}\widehat{\mathbb{J}} \\
\underline{m} \\
\underline{m}\end{array}$ & $\begin{array}{l}\hat{\sigma} \\
\hat{d} \\
\sigma\end{array}$ & 号 & $\begin{array}{l}\hat{I} \\
\stackrel{1}{n} \\
\underline{n}\end{array}$ & $\begin{array}{l}\widehat{\overline{\bar{d}}} \\
0 \\
0 \\
\dot{m}\end{array}$ \\
\hline 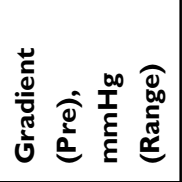 & 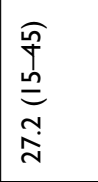 & 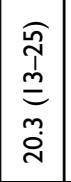 & 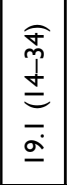 & 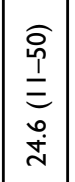 & 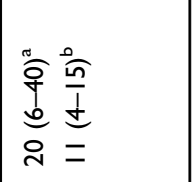 & 足 & 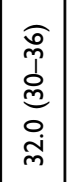 & 竞 & 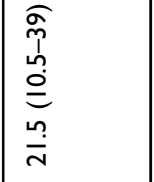 & 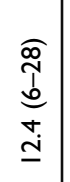 & 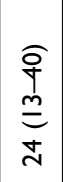 & 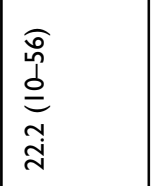 & 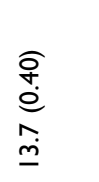 & $\begin{array}{l}\underset{\sigma}{p} \\
\stackrel{1}{=} \\
m \\
\stackrel{0}{0}\end{array}$ \\
\hline 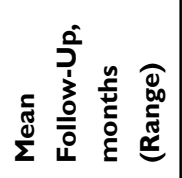 & 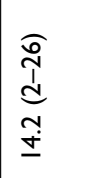 & $\left|\begin{array}{c}\widehat{x} \\
1 \\
\omega \\
n\end{array}\right|$ & 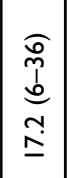 & $\left|\begin{array}{l}\widehat{o} \\
0 \\
1 \\
\hat{n} \\
\end{array}\right|$ & 号 & $\begin{array}{l}\mathfrak{o} \\
d \\
d \\
\stackrel{d}{d}\end{array}$ & $\begin{array}{l}\widehat{\widehat{T}} \\
\stackrel{I}{=} \\
=\end{array}$ & $\frac{\sigma}{d}$ & 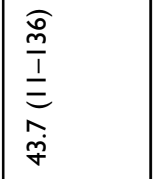 & $\begin{array}{l}\widehat{\sigma} \\
\stackrel{d}{d} \\
o \\
\underline{0}\end{array}$ & \pm & $\ddot{\sim}$ & $\stackrel{\widetilde{f}}{=}$ & 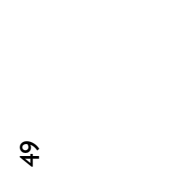 \\
\hline 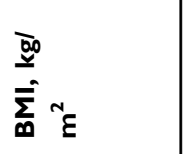 & 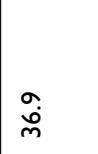 & 号 & $\widehat{\infty}$ & 菂 & 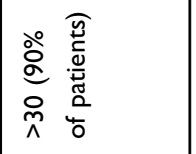 & 号 & $\hat{\sigma}$ & 号 & $\frac{0}{m}$ & $\stackrel{\circ}{\stackrel{్}{~}}$ & $\stackrel{\circ}{\circ}$ & 号 & 品 & $\frac{\sim}{m}$ \\
\hline ப゙ ১゚ & 으 & ำ & 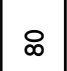 & 으 & 8 & œ & 으 & 号 & $\hat{o}$ & $\alpha$ & 으 & œ & $\cong$ & ০ \\
\hline$\dot{\mathbf{z}}$ & $\simeq$ & $\sigma$ & 으 & 으 & N & $\underline{\underline{n}}$ & $m$ & $\stackrel{\sim}{\sim}$ & $\underline{\infty}$ & $\simeq$ & $\underline{\underline{n}}$ & 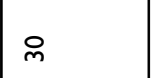 & $\underline{\infty}$ & $\bar{n}$ \\
\hline 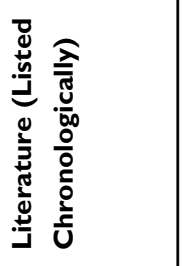 & 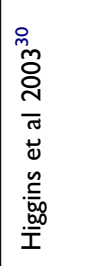 & 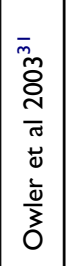 & 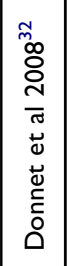 & 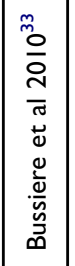 & 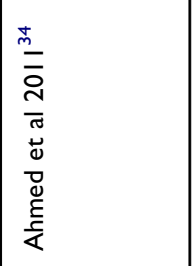 & 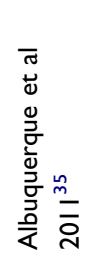 & 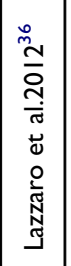 & 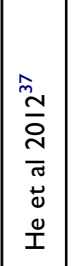 & 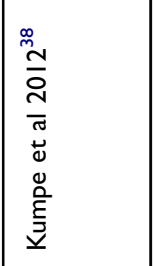 & 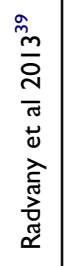 & 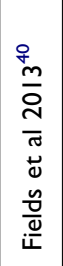 & 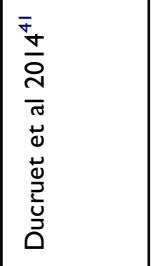 & 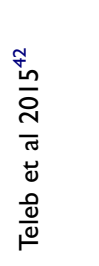 & 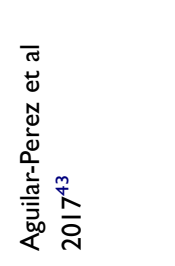 \\
\hline
\end{tabular}




\begin{tabular}{|c|c|c|c|c|c|}
\hline 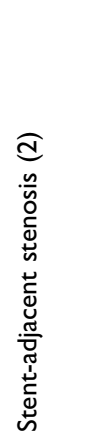 & 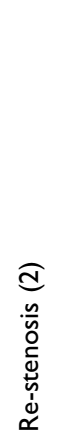 & 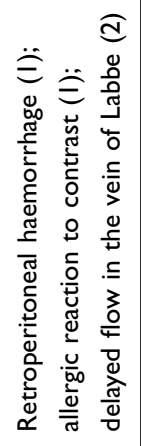 & 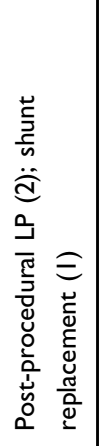 & 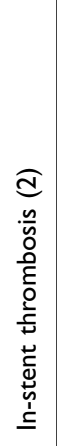 & 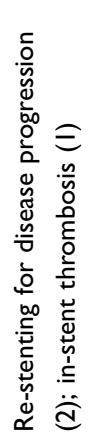 \\
\hline $\begin{array}{l}\widehat{\varrho} \\
\frac{\varrho}{a} \\
\frac{\circ}{a}\end{array}$ & 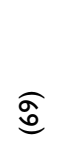 & $\begin{array}{l}\stackrel{\widehat{\infty}}{\stackrel{m}{m}} \\
\stackrel{\Xi}{\Xi}\end{array}$ & $\begin{array}{l}\underset{d}{d} \\
\frac{\infty}{f}\end{array}$ & 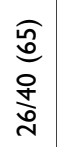 & $\begin{array}{l}\widehat{\widetilde{y}} \\
\underline{y} \\
\underline{\underline{\Phi}}\end{array}$ \\
\hline 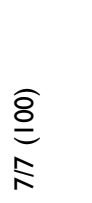 & $\begin{array}{l}\text { ్ㅗ } \\
\text { ָ̃ } \\
\underline{m}\end{array}$ & 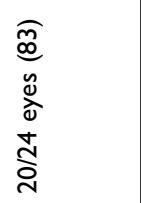 & $\begin{array}{l}\stackrel{\widehat{\varrho}}{\varrho} \\
\stackrel{\infty}{\infty} \\
\underline{\infty}\end{array}$ & $\begin{array}{l}\widehat{\hat{0}} \\
0 \\
\frac{0}{0} \\
\underline{\sigma}\end{array}$ & $\begin{array}{l}\frac{J}{5} \\
\text { o } \\
\frac{d}{d}\end{array}$ \\
\hline $\begin{array}{l}\text { O̊n. } \\
\text { 을 } \\
\text { in }\end{array}$ & $\begin{array}{l}\widehat{\widehat{y}} \\
\underline{\underline{m}} \\
\underline{\underline{\underline{n}}}\end{array}$ & 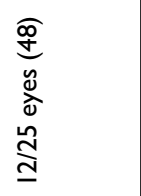 & 号 & $\begin{array}{l}\widehat{\widetilde{c}} \\
\stackrel{\text { ב }}{\underline{\Xi}}\end{array}$ & 号 \\
\hline $\begin{array}{l}\widehat{f} \\
\bar{E} \\
\bar{x}\end{array}$ & 号 & 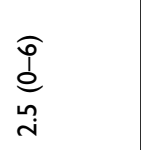 & $\stackrel{\mathscr{N}}{Z}$ & $\frac{\overline{0}}{6}$ & 号 \\
\hline $\begin{array}{l}\widehat{J} \\
\hat{j} \\
\stackrel{5}{0} \\
0 \\
\dot{0} \\
\dot{m}\end{array}$ & \begin{tabular}{l}
$\widehat{o}$ \\
\multirow{1}{1}{} \\
$\hat{\omega}$ \\
$\underline{0}$
\end{tabular} & 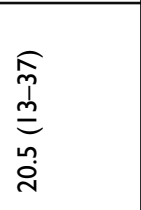 & $\overline{\mathrm{I}}$ & $\stackrel{\underline{n}}{\simeq}$ & 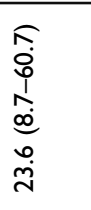 \\
\hline 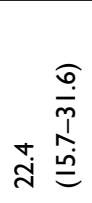 & 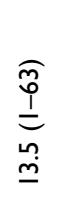 & $\begin{array}{l}\sigma \\
\hat{i} \\
\frac{\hat{q}}{d} \\
\dot{d} \\
\dot{q} \\
\underline{0}\end{array}$ & $\stackrel{t}{\sigma}$ & $\begin{array}{l}\underset{\mathrm{O}}{1} \\
\frac{1}{a} \\
\bar{a}\end{array}$ & 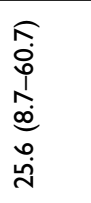 \\
\hline$\frac{\stackrel{L}{q}}{\bar{\sigma}}$ & $\begin{array}{l}\infty \\
\stackrel{m}{m}\end{array}$ & $\stackrel{\widehat{\rho}}{\stackrel{m}{m}}$ & $\stackrel{\text { İ }}{\text { f }}$ & $\stackrel{\alpha}{Z}$ & $\begin{array}{l}\text { Oे } \\
\text { m. }\end{array}$ \\
\hline 8 & $\bar{a}$ & 으 & ळి & 2 & \& \\
\hline 으 & $\Re$ & $\underline{m}$ & $\underline{\infty}$ & $\bar{f}$ & $\mathcal{F}$ \\
\hline 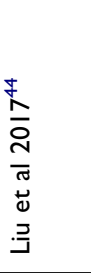 & 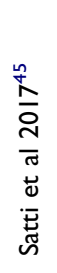 & 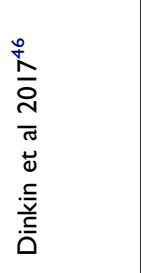 & 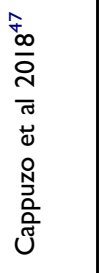 & 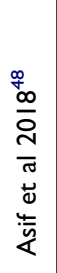 & 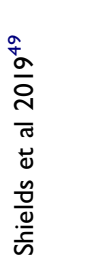 \\
\hline
\end{tabular}

hypertension, stenosis, and trans-stenotic pressure gradient with reduction in ICP. ${ }^{54-56}$ Conflicting evidence of persisting venous sinus stenosis despite normalisation of $\mathrm{ICP}^{57}$ suggests more than one mechanism is at play.

To resolve these seemingly opposing mechanisms, it is now thought that two distinct types of venous sinus stenosis exist in association with IIH:

- The first, thought to occur in the majority of patients, is due to extrinsic compression as a consequence of raised ICP, with a long tapering stenosis and normal arachnoid granulations on neuro-imaging. This type of stenosis is reversible with normalisation of ICP (Figure 1). This has also been termed non-venogenic and is thought to be due to abnormal CSF absorption mechanisms. ${ }^{31}$

- The second, less common type, is due to intrinsic focal venous sinus stenosis (Figure 2), typically due to arachnoid granulation hypertrophy, fibrosis, or deposition. This "venogenic" type may be due to primary venous pathology (such as thrombosis or vasculitis) or anatomical variation. ${ }^{31}$ It is thought that this type may initially be pre-symptomatic, requiring a "second hit" such as weight gain or altered CSF dynamics to result in raised ICP. This is supported by evidence from a study which found bilateral venous sinus stenosis in a group of patients with no papilloedema or symptoms of raised ICP. ${ }^{58}$ This type of stenosis is unresponsive to changes in ICP.

It could be argued that dural venous sinus stenting has no role in a patient with extrinsic venous sinus compression associated with IIH. However, as evidence exists to the contrary, a positive feedback loop, the so-called "collapsible model theory". A mild increase in ICP causes a degree of venous sinus stenosis in a compressible region, resulting in impaired CSF outflow causing a further rise in ICP with more venous sinus compression, and a resultant increase in the trans-stenotic pressure gradient. ${ }^{59}$ This has been likened to a so-called Starling resistor whereby raised ICP restricts venous outflow, maintaining equilibrium between blood inflow and CSF outflow. Dural venous sinus stenting in these patients is theorised to increase vessel rigidity, reduce compression and collapse, and therefore interrupt this positive feedback loop. There is still debate as to what 


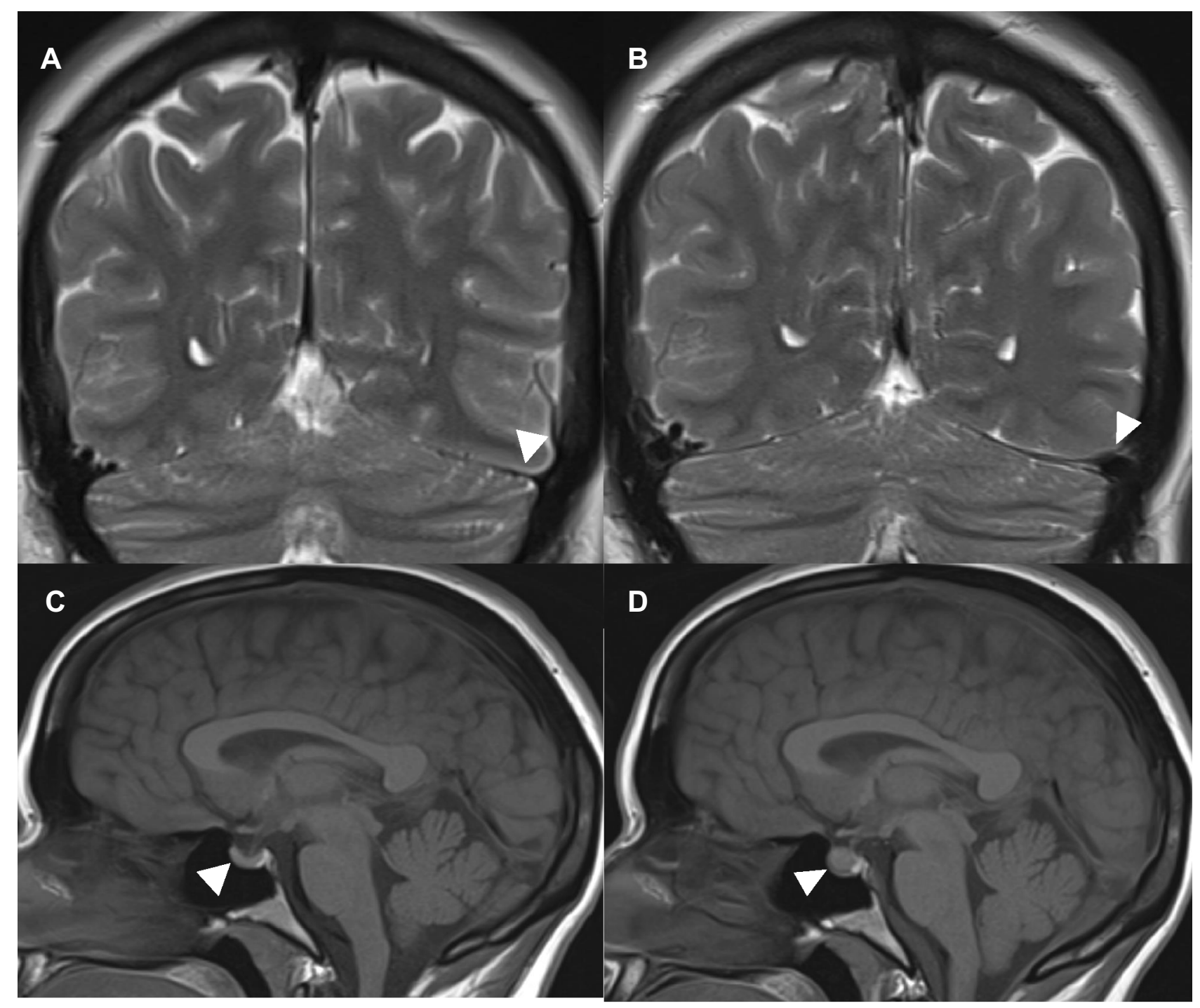

Figure I Radiological changes seen following lumbar puncture in IIH. MRI head imaging coronal T2- weighted image showing improvement in the caliber of the venous sinuses, particularly the left transverse sinus pre-lumbar puncture (A) to post-lumbar puncture (B). MRI head imaging sagittal TI-weighted image showing a partially empty sella (arrow) (C); MRI head sagittal TI-weighted image showing resolution of the partially empty sella following lumbar puncture within I0 days (arrow) (D).

initiates this chain of events, but there is evidence of a positive correlation between BMI, mean intracranial venous pressure, and trans-stenotic pressure gradient in patients with IIH suggesting that weight-gain may be the inciting event. ${ }^{60}$

\section{Role Of Neuro-Imaging In Venous Sinus Stenosis}

The choice of neuro-imaging modality is important both in pre-procedure patient evaluation and post-procedure monitoring for the detection of re-stenosis. By using specific imaging protocols to grade venous sinus stenosis, one study reported a sensitivity and specificity of $93 \%$ for identifying IIH using MRV. ${ }^{50}$ It is recognised that the sensitivity of MRV is dependent on technical factors. In order to achieve an acceptable yield when investigating dural venous sinus pathology, neuroimaging should include the whole area from the SSS to the jugular bulb. There is ongoing research into the role of computerassisted detection to create venous sinus "normograms" in control patients in order to better characterise the sinus changes in patients with suspected IIH. $^{61}$ Other research suggests that time-of-flight MRV is a reliable representation of endoluminal dural venous sinus dimensions, whereas contrast-enhanced MRV tends to overestimate endoluminal sinus dimensions compared with intravascular ultrasound. ${ }^{62}$ Conventional cerebral arteriography is associated with small risk of stroke and has been 


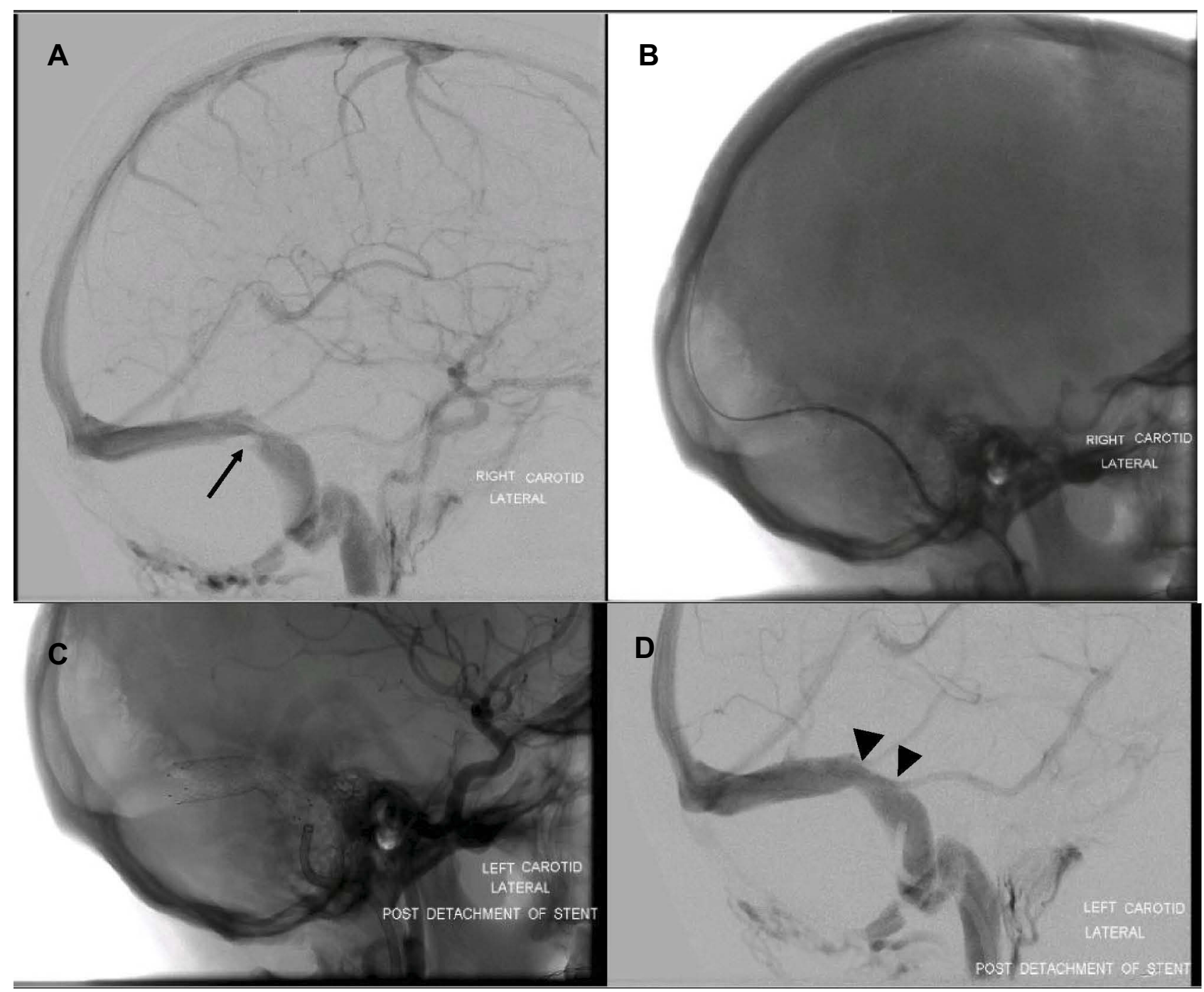

Figure 2 Intrinsic stenosis resolved by stent. This is an example of intrinsic stenosis of the right transverse-sigmoid sinus junction. Angiographic evidence of the stenosis (arrow) (A) followed by imaging during the opening of the stent in the venous sinus (B). Unsubtracted image of the stent released in the sinus (C) followed by evidence of relief of the stenosis and patency of the Labbé vein and the superficial middle cerebral vein (small arrowheads) (D).

shown to miss some venous sinus lesions in patients with $\mathrm{IIH}^{63}$ Direct retrograde cerebral venography (DRCV) is more sensitive and has fewer risks, but alone can also miss lesions. ${ }^{51}$ A gold standard of neuro-imaging has therefore been proposed which combines both DRCV and dural venous manometry. ${ }^{31}$

When it comes to estimating venous sinus pressure gradients, non-invasive imaging is not thought to be suitable for ruling out clinically significant venous sinus stenosis; one study which aimed to correlate angiographic venous sinus measurements to physiological venous outflow obstruction determined by venous manometry, calculated a sensitivity for detecting a significant trans-stenotic pressure gradient with non-invasive magnetic resonance venography (MRV) or computerised tomography venography (CTV) to be $42 \%$, with a negative predictive value of $22 \%{ }^{64}$ This compared unfavourably to invasive imaging modalities such as venous phase arteriography (sensitivity $81 \%$, specificity 91\%) and venography (sensitivity $92 \%$, specificity $73 \%$ ). Intravascular ultrasound provides $360^{\circ}$ visualization with accurate measurements and information regarding the type of stenosis (intrinsic versus extrinsic). ${ }^{46}$

There is evidence that quantitative MRV may be a useful tool in post-stenting surveillance; using this imaging modality, venous outflow increases after venous sinus stenting and correlates with significantly improved sinus venous pressure. ${ }^{65}$ More recently, contrast-enhanced MRV has been shown to be a reliable first-line investigation for monitoring patients following venous sinus stenting, with one group reporting $100 \%$ sensitivity and 100\% negative 
Table 2 Proposed criteria for dural venous sinus stenting in $\mathrm{IIH}$ adapted from Teleb et al $^{42}$

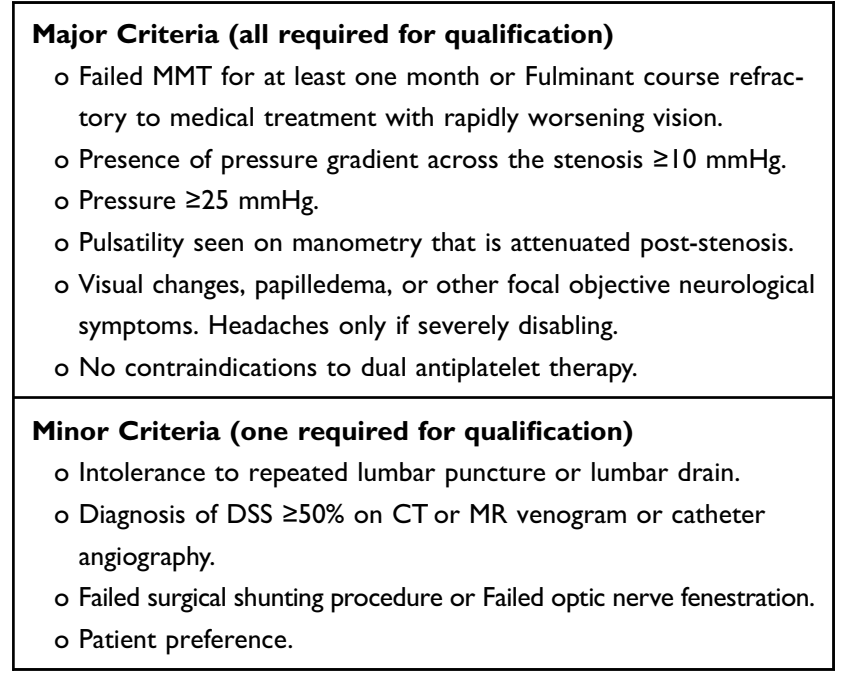

Note: Reproduced from Teleb et al., Stenting and Angioplasty for Idiopathic IntracranialHypertension: A Case Series with Clinical, Angiographic, Ophthalmological, Complication, and Pressure Reporting. John Wiley and Sons. Copyright $\odot 2013$ by the American Society of Neuroimaging.

predictive value for detection of recurrent dural venous sinus stenosis. ${ }^{66}$ The obvious advantage of MRV is that it is non-invasive and avoids exposing patients to ionising radiation or contrast medium. Changes in optical coherence tomography imaging parameters have been shown to correlate with visual improvement, and therefore may be used as an objective, anatomical measure of outcomes in patients following venous sinus stenting. ${ }^{67}$

\section{Establishing A Role Of Venous Sinus Stenting In IIH}

One key question is where does venous stenting sits in the management algorithm for $\mathrm{IIH}$, given that currently there is no class 1 evidence for its use in IIH: for example, should it be reserved for those requiring emergency treatment for fulminant disease? Or those refractory to medical management? As outlined above, it is now accepted that venous sinus stenosis and hypertension may play a role in the pathophysiology of IIH. Therefore, it can be argued that when a dural venous stenosis is identified in a patient and where emergency surgical treatment is required, the most appropriate intervention is one that acts directly on venous sinus haemodynamics, namely venous sinus stenting. They argue that other surgical options such as ONSF and CSF diversion procedures act to reduce CSF pressure; they do not act directly on venous sinus haemodynamics and therefore may not modulate the underlying cause.
Similarly, in deciding on the next course of action for an individual with a suboptimal response to weight loss and medical management, it may be important to establish the degree of venous sinus stenosis. One approach is to perform cerebral digital subtraction venography to establish if a stenotic venous sinus is present with a high trans-stenotic pressure gradient (e.g. $\geq 10 \mathrm{mmHg}$ ). If this is the case, treatment with venous sinus stenting may be appropriate. If stenosis is not present, or the trans-stenotic pressure gradient is sufficiently low to be clinically insignificant, one of the treatments to lower ICP may be more appropriate. One proposed set of criteria for dural venous sinus stenting in IIH is given in Table $2^{42}$ and an overview of the current surgical technique is described by Cappuzzo et al. ${ }^{47}$

\section{Advantages Of Venous Sinus Stenting}

There is growing evidence in the literature of the efficacy of venous sinus stenting. A recent systematic review and meta-analysis identified 473 patients from 24 studies concluded that in patients with refractory IIH and venous sinus stenosis with elevated pressure gradient, venous sinus stenting is associated with a reduction in pressure gradient and ICP, improvement in signs and symptoms of $\mathrm{IIH}$, and acceptable stent survival rates. ${ }^{68}$ Venous sinus stenting appears to be equally effective as both a primary or secondary procedure, following CSF diversion. ${ }^{48}$

Another meta-analysis of 8 studies including 136 patients showed headache improvement in $83 \%$, papilloedema improvement in $97 \%$, and visual acuity improvement in $78 \%$. ${ }^{69}$ Transient visual obscurations and diplopia, when present, resolved in $76 \%$ and $92 \%$, respectively. ${ }^{70}$ The mean pressure gradient reduced from $20.6 \mathrm{mmHg}$ (pre-stent) to 2.7 mmHg (post-stent). ${ }^{69}$ A systematic review comparing headache and visual outcomes in IIH following ONSF, lumboperitoneal shunt (LPS), ventriculoperitoneal shunt (VPS), or venous sinus stenting showed a similar improvement in visual outcomes across all modalities but a modest improvement in headache following CSF diversion in combination with venous sinus stenting. ${ }^{71}$ It is generally accepted however that improvement in headache is not a good proxy for improvement in ICP. There is evidence that venous sinus stenting in the appropriate patient is also associated with a trend towards shorter duration of medical treatment with acetazolamide. ${ }^{72}$ Longer term survival seems to compare favourably with CSF diversion. One study reported 120-day survival of $87.8 \%$ following venous sinus stenting. ${ }^{48}$ Even more convincing was a meta-analysis of 395 patients with a mean follow up of 18.9 months, in which stent survival in $84 \%$ was reported. ${ }^{68}$ 
High BMI has been correlated with worse outcomes in $\mathrm{IIH}$, and there is also a positive correlation between BMI and intracranial venous pressure. Recent evidence suggests venous sinus stenting results in an even greater reduction in venous pressure for these "at risk" patients with higher BMI. ${ }^{60}$ There have been concerns that the effect of venous sinus stenting may not be fast enough to be useful in fulminant IIH. However, there is growing evidence of good efficacy even in patients with acute, severe loss of vision either following high-volume lumbar puncture ${ }^{46}$ or in conjunction with temporary CSF diversion. ${ }^{52}$ This is supported by the results of a review in which only $8 \%$ of patients with papilloedema at presentation developed optic atrophy, suggesting the speed of ICP normalisation was generally sufficient to prevent irreversible damage. ${ }^{70}$ Indeed, venous sinus stenting has been shown to result in immediate alteration of the dural venous sinus pressure measurements in patients with $\mathrm{IIH}^{73}$

\section{Comparing Venous Sinus Stenting To ONSF And CSF Diversion}

The efficacy and safety profile of venous sinus stenting also seems to compare favourably to the other surgical treatment options in IIH, namely ONSF and CSF diversion. ONSF is typically used in patients with medically refractory IIH in whom severe visual loss rather than headache is the primary morbidity or where there is asymmetric papilloedema. The procedure involves creating slits in the optic nerve sheath which are thought to modulate CSF flow either directly via a dural fistula or indirectly by inducing fibrosis and blocking CSF flow between the subarachnoid space around the optic nerve and the intracranial optic nerve. ${ }^{74}$ However, major complications such as orbital or retrobulbar haematoma, orbital cellulitis and traumatic optic neuropathy can occur in $1.5 \%$ of patients. ${ }^{75}$ There is also some evidence of progressive visual decline despite $\mathrm{ONSF}^{75}$ Regarding CSF diversion, a large metaanalysis showed $86 \%$ improvement in headache, $70 \%$ improvement in papilloedema, and more modest improvement in vision. However, in this meta-analysis, there were major complications in $7.6 \%$ of patient, with shunt infection, subdural haematoma, tonsillar herniation, and CSF fistula occurring. ${ }^{69}$ Overall, CSF diversion is associated with significant morbidity and high revision rate ${ }^{27}$ and up to $43 \%$ of patients require additional interventions after CSF diversion, such as shunt revision. ${ }^{1}$

\section{Disadvantages Of Venous Sinus Stenting}

The most common adverse event reported in the literature was transient post-procedure headache, typically lasting days, ipsilateral to the side of stenting which is thought to be secondary to dural stretch. These headaches have been reported in one review to occur in around $30 \%$ of patients. ${ }^{70}$ The rate of serious complications (such as intracranial haemorrhage and venous sinus thrombosis) has been reported as less than $2 \%$ in a number of systematic reviews ${ }^{68,76}$ and around $5 \%$ in a large case series. ${ }^{48}$ Serious complications following venous sinus stenting include subdural haemorrhage, ${ }^{34}$ intracranial dural arteriovenous fistula formation, ${ }^{77,78}$ occlusion of the contralateral transverse sinus $^{79}$ severe cerebellar haemorrhage. ${ }^{80}$ Impaired drainage of the ipsilateral vein of Labbé (VOL) has a reported incidence of $13 \%$ in one study. ${ }^{81}$ This is thought to occur when the stent is placed across the transverse and sigmoid sinuses, covering the VOL ostium with potentially severe consequences including cerebral oedema, venous cerebral ischaemia, and cerebral haemorrhage. Procedure-related complications include femoral pseudo-aneurysm, contrast extravasation, and anaphylaxis. In relation to potential thrombotic complications of venous sinus stenting, there is considerable variation in the use of antiplatelets and anticoagulants before and after venous sinus stenting. Most commonly, dual anti-platelet therapy (with aspirin and clopidogrel) is started as a pre-procedure and continued for at least 6 months to allow for epithelialisation of the stent to occur. Further research in this area will hopefully lead to a more standardised approach, and a reduction in the rate of thrombotic complications. In one review, the overall rate of serious complication following venous sinus stenting $(2.9 \%)$ was higher than following ONSF (1.5\%) but significantly lower than following CSF diversion $(7.6 \%){ }^{69}$

The most common cause of failure is re-stenosis, which can occur in or adjacent to an existing stent. In-stent thrombosis may occur secondary to suboptimal placement of the original stent with residual thrombosis proximal to the stent. ${ }^{49}$ The mechanism of stent-adjacent stenosis (SAS) is poorly understood, but it is recognised as the main reason for stent revision. Postulated mechanisms are raised ICP or pressure gradient, floppy sinuses, uncontrolled endothelialisation, or ongoing extrinsic compression beyond the stented area which continues to incite the pathological positive feedback loop. ${ }^{68}$ This last point is supported by evidence from Ahmed et $\mathrm{al}^{34}$ where 5 of the 6 patients who 
required re-stenting had the long tapering stenosis and all of these had SAS. The pattern of change in the trans-stenosis venous pressure gradient may be predictive of SAS. ${ }^{82}$ The rate of re-stenosis varies in the literature. A meta-analysis of 395 patients with a mean follow-up of 18.9 months estimated the rate of SAS to be $14 \% .{ }^{68}$ Asif et al ${ }^{48}$ reported the re-stenosis rate to be at least $20 \%$ at 120 days. Satti et al ${ }^{69}$ reported the rate of repeat procedures for re-stenosis to be $10.3 \%$, with conversion to CSF diversion in $2.2 \%$.

Research into the potential predictors for re-treatment has shown possible evidence that raised BMI and AfricanAmerican race is associated with higher retreatment rates. $^{83}$ Haemodynamic failure was strongly associated with female gender and pure extrinsic compression of the transverse-sigmoid junction. ${ }^{84}$ Patient presenting with highly raised opening pressures and those with persisting papilloedema post-procedure are at increased risk of stent failure. ${ }^{85}$ It is thought there may be a subgroup of IIH patients who are refractory to venous sinus stenting, in whom permanent CSF diversion is still required for disease control.

\section{Conclusion}

Our knowledge of IIH is advancing rapidly and a modern approach to the disease entity is emerging. ${ }^{7,9,86}$ Interventions for those who have precipitous visual loss needs to be investigated in a systematic way to provide a high level of confidence that vision will be preserved or improved. It seems apparent that venous sinus stenting does have a role in the management of IIH. However, clear study outcomes are required to systematically study its placement, and as such as validated trial outcomes are now required which should include both headache and visual outcomes. Although traditionally the primary surgical treatment of medically refractory IIH has been with CSF diversion, the efficacy and low complication rate associated with dural venous sinus stenting is appealing. As the evidence for venous sinus stenting is so far based on retrospective or uncontrolled prospective studies, there is a need for a more robust evidence base to inform practice in this area. Prospective head-to-head trials such as the VISION trial ${ }^{87}$ comparing venous sinus stenting versus CSF diversion procedure will be of great benefit. Despite good evidence of the effect of stenting on the venous sinus pressure gradient, the effect on CSF pressure is less clearly understood. Recent evidence however shows a significant decrease in CSF opening pressure that is maintained at 3 months post procedure, independent of weight loss or medical therapy. ${ }^{88}$ Unfortunately, CSF pressure post-stenting and at subsequent follow-up is often not reported in published studies. Further studies specifically measuring CSF pressure before and after stenting will improve our knowledge of this relationship. In order to compare the results from future studies, some authors have suggested angiography to confirm stenosis and pressure gradient. ${ }^{69}$ There needs to be standardised collection of data relating to patient characteristics, preand post-stenting headache metrics, neuro-ophthalmic visual examination, CSF studies, and quality of life measures. Long-term follow-up of clinical features and stent patency also need to be reported.

\section{Disclosure}

Dr Allan Thomas report personal fees from MicroVention, outside the submitted work; Miss Susan Mollan reports personal fees from Roche, Santen, Santhera, Allergan, Heidelberg Engineering, and Neurodiem, outside the submitted work. The authors report no other conflicts of interest in this work.

\section{References}

1. Mollan SP, Ali F, Hassan-Smith G, et al. Evolving evidence in adult idiopathic intracranial hypertension: pathophysiology and management. J Neurol Neurosurg Psychiatry. 2016;87(9):982-992. doi:10.1136/jnnp2015-311302

2. Friedman DI, Liu GT, Digre KB. Revised diagnostic criteria for the pseudotumor cerebri syndrome in adults and children. Neurology. 2013;81:1159-1165. doi:10.1212/WNL.0b013e3182a55f17

3. Raoof N, Sharrack B, Pepper IM, Hickman SJ. The incidence and prevalence of idiopathic intracranial hypertension in Sheffield, UK. Eur J Neurol. 2011;18:1266-1268. doi:10.1111/ene.2011.18.issue-10

4. Mollan SP, Aguiar M, Evison F, et al. The expanding burden of idiopathic intracranial hypertension. Eye. 2018;81:1159.

5. Markey KA, Mollan SP, Jensen RH, Sinclair AJ. Understanding idiopathic intracranial hypertension: mechanisms, management, and future directions. Lancet Neurol. 2016;15(1):78-79. doi:10.1016/ S1474-4422(15)00298-7

6. Hornby C, Botfield H, O'Reilly MW, et al. Evaluating the fat distribution in idiopathic intracranial hypertension using dual-energy X-ray absorptiometry scanning. Neuroophthalmology. 2017;42 (2):99-104. doi:10.1080/01658107.2017.1334218

7. Hornby C, Mollan SP, Botfield H, et al. Metabolic concepts in idiopathic intracranial hypertension and their potential for therapeutic intervention. J Neuroophthalmol. 2018;38(4):522-530. doi:10.1097/ WNO.0000000000000684

8. O'Reilly MW, Westgate CS, Hornby C, et al. A unique androgen excess signature in idiopathic intracranial hypertension is linked to cerebrospinal fluid dynamics. JCI Insight. 2019;4(6):125348. doi:10.1172/jci.insight. 122686

9. Adderley NJ, Subramanian A, Nirantharakumar K, et al. Association between idiopathic intracranial hypertension and risk of cardiovascular diseases in women in the United Kingdom. JAMA Neurol. 2019;8: e191812.

10. Mollan SP, Spitzer D, Nicholl DJ. Raised intracranial pressure in those presenting with headache. BMJ. 2018;363:k3252. doi:10.1136/ bmj.k3252 
11. Mollan SP, Hoffman J, Sinclair AJ. Advances in the understanding of headache in idiopathic intracranial hypertension. Curr Opin Neurol. 2019;32(1):92-98. doi:10.1097/WCO.0000000000000651

12. Mollan SP, Markey KA, Benzimra JD, et al. A practical approach to, diagnosis, assessment and management of idiopathic intracranial hypertension. Pract Neurol. 2014;14(6):380-390. doi:10.1136/practneurol-2014-000821

13. Corbett JJ, Savino PJ, Thompson HS, et al. Visual loss in pseudotumor cerebri. Follow-up of 57 patients from five to 41 years and a profile of 14 patients with permanent severe visual loss. Arch Neurol. 1982;39:461-474. doi:10.1001/archneur.1982.00510200003001

14. Mulla Y, Markey KA, Woolley RL, et al. Headache determines quality of life in idiopathic intracranial hypertension. J Headache Pain. 2015;16:521. doi:10.1186/s10194-015-0521-9

15. Scotton WJ, Mollan SP, Walters T, et al. Characterising the patient experience of diagnostic lumbar puncture in idiopathic intracranial hypertension: a cross-sectional online survey. BMJ Open. 2018;8(5): e020445. doi:10.1136/bmjopen-2017-020445

16. Mollan SP, Davies B, Silver NC, et al. Idiopathic intracranial hypertension: consensus guidelines on management. J Neurol Neurosurg Psychiatry. 2018;89(10):1088-1100. doi:10.1136/jnnp2017-317440

17. Mollan SP, Hemmings K, Herd C, et al. What are the research priorities for idiopathic intracranial hypertension? A priority setting partnership between patients and health care professionals. BMJ Open. 2019;9(3):e026573. doi:10.1136/bmjopen-2018-026573

18. Piper R, Kalyvas AV, Young AMH, et al. Interventions for idiopathic intracranial hypertension. Cochrane Database Syst Rev. 2015;8: CD003434.

19. Ball AK, Howman A, Wheatley K, et al. A randomised controlled trial of treatment for idiopathic intracranial hypertension. J Neuro.l. 2011;258:874-881. doi:10.1007/s00415-010-5861-4

20. Wall M, McDermott MP, Kieburtz KD, et al. Effect of acetazolamide on visual function in patients with idiopathic intracranial hypertension: the idiopathic intracranial hypertension treatment trial. JAMA. 2014;311:1641-1651. doi:10.1001/jama.2014.3312

21. Sinclair AJ, Burdon MA, Nightingale PG, et al. Low energy diet and intracranial pressure in women with idiopathic intracranial hypertension: prospective cohort study. BMJ. 2010;341:c2701-c2701. doi:10.1136/bmj.c2701

22. Ottridge R, Mollan SP, Botfield H, et al. Randomised controlled trial of bariatric surgery versus a community weight loss programme for the sustained treatment of idiopathic intracranial hypertension: the Idiopathic Intracranial Hypertension Weight Trial (IIH:WT) protocol. BMJ Open. 2017;7(9):e017426. doi:10.1136/bmjopen-2017-017426

23. Çelebisoy N, Gökçay F, Şirin H, Akyürekli Ö. Treatment of idiopathic intracranial hypertension: topiramate vs acetazolamide, an open-label study. Acta Neurol Scand. 2007;116(5):322-327. doi:10.1111/ane.2007.116.issue-5

24. Scotton WJ, Botfield HF, Westgate CS, et al. Topiramate is more effective than acetazolamide at lowering intracranial pressure Cephalalgia. 2019;39(2):209-218. doi:10.1177/0333102418776455

25. Markey K, Mitchell J, Botfield H, et al. 11 $\beta$-hydroxysteroid dehydrogenase type 1 inhibition in idiopathic intracranial hypertension: a double-blind randomized controlled trial. Brain Commun. 2019. in progress.

26. Hoffmann J, Mollan SP, Paemeleire K, et al. European headache federation guideline on idiopathic intracranial hypertension. J Headache Pain. 2018;19(1):93. doi:10.1186/s10194-018-0919-2

27. Sinclair AJ, Kuruvath S, Sen D, et al. Is cerebrospinal fluid shunting in idiopathic intracranial hypertension worthwhile? A 10-year review. Cephalalgia. 2011;31:1627-1633. doi:10.1177/0333102411423305

28. Kalyvas AV, Hughes M, Koutsarnakis C, et al. Efficacy, complications and cost of surgical interventions for idiopathic intracranial hypertension: a systematic review of the literature. Acta Neurochir (Wien.). 2017;159(1):33-49. doi:10.1007/s00701-016-3010-2
29. Gilbert AL, Chwalisz B, Mallery R. Complications of optic nerve sheath fenestration as a treatment for idiopathic intracranial hypertension. Semin Ophthalmol Y. 2018;33(1):36-41. doi:10.1080/ 08820538.2017.1353810

30. Higgins JN, Owler BK, Cousins C, et al. Venous sinus stenting for refractory benign intracranial hypertension. Lancet. 2002;359:228-230. doi:10.1016/S0140-6736(02)07440-8

31. Owler BK, Parker G, Halmagyi GM, et al. Pseudotumor cerebri syndrome: venous sinus obstruction and its treatment with stent placement. J Neurosurg. 2003;98(5):1045-1055. doi:10.3171/jns.2003.98.5.1045

32. Donnet A, Metellus P, Levrier O, et al. Endovascular treatment of idiopathic intracranial hypertension: clinical and radiologic outcome of 10 consecutive patients. Neurology. 2008;70:641-647. doi:10.1212/01. wnl.0000299894.30700.d2

33. Bussière M, Falero R, Nicolle D, et al. Unilateral transverse sinus stenting of patients with idiopathic intracranial hypertension. $\mathrm{Am}$ J Neuroradiol. 2010;31:645-650. doi:10.3174/ajnr.A1890

34. Ahmed RM, Wilkinson M, Parker GD, et al. Transverse sinus stenting for idiopathic intracranial hypertension: a review of 52 patients and of model predictions. Am J Neuroradiol. 2011;32:1408-1414. doi:10.3174/ajnr.A2575

35. Albuquerque FC, Dashti SR, Hu YC, et al. Intracranial venous sinus stenting for benign intracranial hypertension: clinical indications, technique, and preliminary results. World Neurosurg. 2011;75:648-652. doi:10.1016/j.wneu.2010.11.012

36. Lazzaro MA, Darkhabani Z, Remler BF, et al. Venous sinus pulsatility and the potential role of dural incompetence in idiopathic intracranial hypertension. Neurosurgery. 2012;71(4):877-884. doi:10.1227/NEU. 0b013e318267a8f9

37. He C-ZC, Ji X-MX, Wang L-JL, et al. Endovascular treatment for venous sinus stenosis in idiopathic intracranial hypertension. Zhonghua Yi Xue Za Zhi. 2012;92(11):748-751.

38. Kumpe DA, Bennett JL, Seinfeld J, et al. Dural sinus stent placement for idiopathic intracranial hypertension. J Neurosurg. 2012;116:538-548. doi:10.3171/2011.10.JNS101410

39. Radvany MG, Solomon D, Nijjar S, et al. Visual and neurological outcomes following endovascular stenting for pseudotumor cerebri associated with transverse sinus stenosis. $J$ Neuroophthalmol. 2013;33:117-122. doi:10.1097/WNO.0b013e31827f18eb

40. Fields JD, Javedani PP, Falardeau J, et al. Dural venous sinus angioplasty and stenting for the treatment of idiopathic intracranial hypertension. J Neurointerv Surg. 2013;5:62-68. doi:10.1136/neurintsurg-2011-010156

41. Ducruet AF, Crowley RW, McDougall CG, Albuquerque FC. Longterm patency of venous sinus stents for idiopathic intracranial hypertension. J Neurointerv Surg. 2014;6:238-242. doi:10.1136/neurintsurg-2013-010691

42. Teleb MS, Cziep ME, Issa M, et al. Stenting and angioplasty for idiopathic intracranial hypertension: a case series with clinical, angiographic, ophthalmological, complication, and pressure reporting. J Neuroimaging. 2015;25:72-80. doi:10.1111/jon.2015. 25.issue-1

43. Aguilar-Perez M, Martinez-Moreno R, Kurre W, et al. Endovascular treatment of idiopathic intracranial hypertension: retrospective analysis of immediate and long-term results in 51 patients. Neuroradiology. 2017;59:277-287. doi:10.1007/s00234-017-1783-5

44. Liu KC, Starke RM, Durst CR, et al. Venous sinus stenting for reduction of intracranial pressure in IIH: a prospective pilot study. J Neurosurg. 2017;127:1126-1133. doi:10.3171/2016.8.JNS16879

45. Satti SR, Leishangthem L, Spiotta A, Chaudry MI. Dural venous sinus stenting for medically and surgically refractory idiopathic intracranial hypertension. Interv Neuroradiol. 2017;23:186-193. doi:10. $1177 / 1591019916680110$

46. Dinkin MJ, Patsalides A. Venous sinus stenting in idiopathic intracranial hypertension: results of a prospective trial. J Neuroophthalmol. 2017;37 (2):113-121. doi:10.1097/WNO.0000000000000426 
47. Cappuzzo JM, Hess RM, Morrison JF, et al. Transverse venous stenting for the treatment of idiopathic intracranial hypertension, or pseudotumor cerebri. Neurosurg Focus. 2018;45(1):E11. doi:10.3171/2018.5.FOCUS18102

48. Asif H, Craven CL, Siddiqui AH, et al. Idiopathic intracranial hypertension: 120-day clinical, radiological, and manometric outcomes after stent insertion into the dural venous sinus. J Neurosurg. 2018;129:723-731. doi:10.3171/2017.4.JNS162871

49. Shields LBE, Shields CB, Yao TL, et al. Endovascular treatment for venous sinus stenosis in idiopathic intracranial hypertension: an observational study of clinical indications, surgical technique, and long-term outcomes. World Neurosurg. 2019;121:e165-e171. doi:10.1016/j.wneu.2018.09.070

50. Farb RI, Vanek I, Scott JN, et al. Idiopathic intracranial hypertension: the prevalence and morphology of sinovenous stenosis. Neurology. 2003;60(9):1418-1424. doi:10.1212/01.WNL.0000066683.34093.E2

51. King JO, Mitchell PJ, Thomson KR, Tress BM. Cerebral venography and manometry in idiopathic intracranial hypertension. Neurology. 1995;45(12):2224-2228. doi:10.1212/WNL.45.12.2224

52. Elder BD, Rory Goodwin C, Kosztowski TA, et al. Venous sinus stenting is a valuable treatment for fulminant idiopathic intracranial hypertension. J Clin Neurosci. 2015;22:685-689. doi:10.1016/j. jocn.2014.10.012

53. Davson H, Hollingsworth M, Segal MB. The mechanism and drainage of the cerebrospinal fluid. Brain. 1973;93:665-678. doi:10.10 93/brain/93.4.665

54. King JO, Mitchell PJ, Thompson KR, Tress BM. Manometry combined with cervical puncture in idiopathic intracranial hypertension. Neurology. 2002;58:26-30. doi:10.1212/WNL.58.1.26

55. Rohr A, Dörner L, Stingele R, et al. Reversibility of venous sinus obstruction in idiopathic intracranial hypertension. Am J Neuroradiol. 2007;28(4):656-659.

56. Buell TJ, Raper DMS, Pomeraniec IJ, et al. Transient resolution of venous sinus stenosis after high-volume lumbar puncture in a patient with idiopathic intracranial hypertension. J Neurosurg. 2018;129 (1):153-156. doi:10.3171/2017.3.JNS163181

57. Bono F, Giliberto C, Mastrandrea C, et al. Transverse sinus stenoses persist after normalization of the CSF pressure in IIH. Neurology. 2005;65(7):1090-1093. doi:10.1212/01.wnl.0000178889.63571.e5

58. Kelly LP, Saindane AM, Bruce BB, et al. Does bilateral transverse cerebral venous sinus stenosis exist in patients without increased intracranial pressure? Clin Neurol Neurosurg. 2013;115(8):1215-1219. doi:10.1016/j.clineuro.2012.11.004

59. Stevens SA, Previte M, Lakin WD, et al. Idiopathic intracranial hypertension and transverse sinus stenosis: a modelling study. Math Med Biol. 2007;24:85-109. doi:10.1093/imammb/dq1025

60. Raper DMS, Ding D, Buell TJ, et al. Effect of body mass index on venous sinus pressures in idiopathic intracranial hypertension patients before and after endovascular stenting. Neurosurgery. 2018;82 (4):555-561. doi:10.1093/neuros/nyx186

61. Anconina R, Zur D, Kesler A, et al. Creating normograms of dural sinuses in healthy persons using computer-assisted detection for analysis and comparison of cross-section dural sinuses in the brain. J Clin Neurosci. 2017;40:190-194. doi:10.1016/j.jocn.2017.02.006

62. Boddu SR, Gobin P, Oliveira C, et al. Anatomic measurements of cerebral venous sinuses in idiopathic intracranial hypertension patients. PLoS One. 2018;13(6):e0196275. doi:10.1371/journal. pone. 0196275

63. Karahalios DG, Rekate HL, Khayata MH, et al. Elevated intracranial venous pressure as a universal mechanism in pseudotumor cerebri of varying etiologies. Neurology. 1996;46:198-202. doi:10.1212/ WNL.46.1.198

64. West JL, Greeneway GP, Garner RM, et al. Correlation between angiographic stenosis and physiologic venous sinus outflow obstruction in idiopathic intracranial hypertension. J Neurointerv Surg. 2019;11(1):90-94. doi:10.1136/neurintsurg-2018-014004
65. Esfahani DR, Stevenson M, Moss HE, et al. Quantitative magnetic resonance venography is correlated with intravenous pressures before and after venous sinus stenting. Neurosurgery. 2015;77:254-260. doi:10.1227/NEU.0000000000000771

66. Boddu SR, Gobin P, Oliveira C, et al. Contrast enhanced magnetic resonance venography in the follow-up evaluation of idiopathic intracranial hypertension patients with cerebral venous sinus stenting. Clin Imaging. 2018;50:330-335. doi:10.1016/j.clinimag. 2018.04.016

67. Smith KA, Peterson JC, Arnold PM, et al. A case series of dural venous sinus stenting in idiopathic intracranial hypertension: association of outcomes with optical coherence tomography. Int J Neurosci. 2017;127:145-153. doi:10.3109/00207454.2016.1152 967

68. Saber H, Lewis W, Sadeghi M, et al. Stent survival and stent-adjacent stenosis rates following venous sinus stenting for idiopathic intracranial hypertension: a systematic review and meta-analysis. Interv Neurol. 2018;7(6):490-500. doi:10.1159/000490578

69. Satti SR, Leishangthem L, Chaudry MI. Meta-analysis of CSF diversion procedures and dural venous sinus stenting in the setting of medically refractory idiopathic intracranial hypertension. Am J Neuroradiol. 2015;36(10):1899-1904. doi:10.3174/ajnr.A4377

70. Dinkin MJ, Patsalides A. Venous sinus stenting for idiopathic intracranial hypertension: where are we now? Neurol Clin. 2017;35:59-81. doi:10.1016/j.ncl.2016.08.006

71. Lai LT, Danesh-Meyer HV, Kaye AH. Visual outcomes and headache following interventions for idiopathic intracranial hypertension. $J$ Clin Neurosci. 2014;21:1670-1678. doi:10.1016/j.jocn.2014.02.0 25

72. Shazly TA, Jadhav AP, Aghaebrahim A, et al. Venous sinus stenting shortens the duration of medical therapy for increased intracranial pressure secondary to venous sinus stenosis. J Neurointerv Surg. 2018;10:310-314. doi:10.1136/neurintsurg-2017-013103

73. Boddu SR, Gobin P, Oliveira C, Dinkin M, Patsalides A. Pressure variations in cerebral venous sinuses of idiopathic intracranial hypertension patients. J Vasc Interv Neurol. 2018;10(1):25-30.

74. Banta JT, Farris BK. Pseudotumor cerebri and optic nerve sheath decompression. Ophthalmology. 2000;107:1907-1912. doi:10.1016/ S0161-6420(00)00340-7

75. Uretsky S. Surgical interventions for idiopathic intracranial hypertension. Curr Opin Ophthalmol. 2009;20:451-455. doi:10.1097/ ICU.0b013e3283313c1c

76. Nicholson P, Brinjikji W, Radovanovic I, et al. Venous sinus stenting for idiopathic intracranial hypertension: a systematic review and meta-analysis. J Neurointerv Surg. 2018. doi:10.1136/neurintsurg-2018014172

77. Li K, Ren M, Meng R, Wang F, Ji X. Dural arteriovenous fistula formation complicated cerebral venous sinus stenosis after venous sinus stenting. World Neurosurg. 2018;120:400-402. doi:10.1016/j. wneu.2018.08.230

78. Buell TJ, Raper DM, Ding D, et al. Development of an intracranial dural arteriovenous fistula after venous sinus stenting for idiopathic intracranial hypertension. J Neurointerv Surg. 2018;10(7):e15. doi:10.1136/neurintsurg-2017-013282.rep

79. Coffman SA, Singh J, Wolfe S, Fargen KM. Unexpected occlusion of the contralateral transverse sinus after stenting for idiopathic intracranial hypertension. Interv Neuroradiol. 2018;24(6):718-721. doi:10.1177/1591019918787161

80. Lavoie P, Audet MÈ, Gariepy JL, et al. Severe cerebellar hemorrhage following transverse sinus stenting for idiopathic intracranial hypertension. Interv Neuroradiol. 2018;24(1):100-105. doi:10.1177/ 1591019917734389

81. Boddu SR, Gobin P, Dinkin M, et al. Impaired drainage of vein of Labbé following venous sinus stenting for idiopathic intracranial hypertension. J Neurointerv Surg. 2018;11:1-7. 
82. Raper D, Buell TJ, Ding D, et al. Pattern of pressure gradient alterations after venous sinus stenting for idiopathic intracranial hypertension predicts stent-adjacent stenosis: a proposed classification system. J Neurointerv Surg. 2018;10(4):391-395. doi:10.1136/neurintsurg-2017013135

83. El Mekabaty A, Obuchowski NA, Luciano MG, et al. Predictors for venous sinus stent retreatment in patients with idiopathic intracranial hypertension. J Neurointerv Surg. 2017;9(12):1228-1232.

84. Kumpe DA, Seinfeld J, Huang X, et al. Dural sinus stenting for idiopathic intracranial hypertension: factors associated with hemodynamic failure and management with extended stenting. $J$ Neurointerv Surg. 2016;9:867-874. doi:10.1136/neurintsurg-201 6-012810
85. Goodwin CR, Elder BD, Ward A, et al. Risk factors for failed transverse sinus stenting in pseudotumor cerebri patients. Clin Neurol Neurosurg. 2014;127:75-78. doi:10.1016/j.clineuro.2014.09.015

86. Mitchell J, Mollan SP, Vivek V, Sinclair AJ. Novel advances in monitoring and therapeutic approaches in IIH. Curr Opin Neurol. 2019;32(3):422-443. doi:10.1097/WCO.0000000000000690

87. Chandran A, Pulhorn H, McMahon C. Idiopathic intracranial hypertension VISION (Venous intervention versus shunting in IIH for optic nerve swelling) trial: patient perspective questionnaire. $\mathrm{Br} J$ Neurosurg. 2017;21:1-5

88. Patsalides A, Oliveira C, Wilcox J, et al. Venous sinus stenting lowers the intracranial pressure in patients with idiopathic intracranial hypertension. J Neurointerv Surg. 2018. doi:10.1136/neurintsurg-2018-014032
Eye and Brain

\section{Publish your work in this journal}

Eye and Brain is an international, peer-reviewed, open access journal focusing on clinical and experimental research in the field of neuroophthalmology. All aspects of patient care are addressed within the journal as well as basic research. Papers covering original research basic science, clinical and epidemiological studies, reviews and

Submit your manuscript here: https://www.dovepress.com/eye-and-brain-journal
Dovepress

evaluations, guidelines, expert opinion and commentary, case reports and extended reports are welcome. The manuscript management system is completely online and includes a very quick and fair peerreview system, which is all easy to use. Visit http://www.dovepress. com/testimonials.php to read real quotes from published authors. 